INPLASY

PROTOCOL

To cite: Bai et al. Predictive model for the probability of malignancy in solitary pulmonary nodules: A metaanalysis. Inplasy protocol 2021100006. doi:

10.37766/inplasy2021.10.0006

Received: 02 October 2021

Published: 02 October 2021

Corresponding author:

Li-Juan Wen

2095445490@qq.com

Author Affiliation:

The third affiliated hospital of Qiqihar Medical University.

Support: None.

Review Stage at time of this submission: Preliminary searches.

Conflicts of interest:

None declared.

\section{Predictive model for the probability of malignancy in solitary pulmonary nodules: A meta-analysis}

Review question / Objective: We conducted a meta-analysis to evaluate the diagnostic ability of predictive model in differential diagnosis of solitary pulmonary nodules.

Information sources: Extracted data and study details were as follows: first authors' name, year of publication, publication countries or areas, types of design, blinding status, sample size, SPN size, reference standards, contents of predictive models, and true-positive (TP), false-positive (FP), truenegative (TN), and false-negative (FN) results.

Main outcome(s): Sensitivity, specificity, positive likelihood ratio (PLR), negative likelihood ratio (NLR), diagnostic score, and summary receiver operating characteristic (SROC) curve.

INPLASY registration number: This protocol was registered with the International Platform of Registered Systematic Review and Meta-Analysis Protocols (INPLASY) on 02 October 2021 and was last updated on 02 October 2021 (registration number INPLASY2021100006).

\section{INTRODUCTION}

Review question / Objective: We conducted a meta-analysis to evaluate the diagnostic ability of predictive model in differential diagnosis of solitary pulmonary nodules.

Condition being studied: At present, solitary pulmonary nodules (SPNs) are commonly detected by computed tomography (CT) because the thoracic CT screening has been used as a routine physical examination item. SPNs may be malignant and represent early stage lung cancer. Approximate $55 \%-77 \%$ of SPNs are malignant and the probability of malignancy increases as the diameter of SPN increases. Although pathological 
diagnosis is the final diagnostic approach for the SPNs, the samples should be obtained via the invasive approaches (biopsy or surgical resection). To avoid the unnecessary invasive diagnostic approaches as far as possible, a comprehensive assessment for SPNs is necessary. Differential diagnosis of SPNs cannot be done by assessing only one aspect of clinical or radiological features. Therefore, establishing the predictive model for the probability of malignancy in SPNs is necessary. The first predictive model of SPNs was reported by Mayo clinic at 1999. Afterwards, many predictive models have been created from many countries. The sensitivity and specificity values of these models varied a lot from different studies. The differences may be attributed to the sample size, whether the models contained the positron emission tomography (PET)/CT or tumor marker tests. As such, there is a clear need for large-scale studies with larger datasets in order to more accurately evaluate the diagnostic ability of these models.

\section{METHODS}

Search strategy: $((()($ diagnosis [Title) Abstract]) OR (analysis[Title/Abstract])) OR (probability[Title/Abstract])) OR (differential[Title/Abstract])) OR (predictive[Title/Abstract])) AND (model[Title/Abstract])) AND (((pulmonary nodule[Title/Abstract]) OR (Iung nodule[Title/Abstract])) OR (SPN[Title/ Abstract])).

Participant or population: SPN patients.

Intervention: Malignant SPN.

Comparator: Benign SPN.

Study designs to be included: The inclusion criteria were: (a) studies regarding of differential diagnosis of malignant and benign SPNs; (b) SPN lesion $\leq 30 \mathrm{~mm}$; (c) studies which provided the predictive models; (d) studies which provided the sensitivity and specificity values. The exclusion criteria were: (a) non-human studies; (b) review studies; and (c) case reports.

Eligibility criteria: The inclusion criteria were: (a) studies regarding of differential diagnosis of malignant and benign SPNs; (b) SPN lesion $\leq 30 \mathrm{~mm}$; (c) studies which provided the predictive models; (d) studies which provided the sensitivity and specificity values. The exclusion criteria were: (a) non-human studies; (b) review studies; and (c) case reports.

Information sources: Extracted data and study details were as follows: first authors' name, year of publication, publication countries or areas, types of design, blinding status, sample size, SPN size, reference standards, contents of predictive models, and true-positive (TP), falsepositive (FP), true-negative (TN), and falsenegative (FN) results.

Main outcome(s): Sensitivity, specificity, positive likelihood ratio (PLR), negative likelihood ratio (NLR), diagnostic score, and summary receiver operating characteristic (SROC) curve.

Quality assessment / Risk of bias analysis: The sensitivity, specificity, positive likelihood ratio (PLR), negative likelihood ratio (NLR), diagnostic score, and summary receiver operating characteristic (SROC) curve were pooled by the Stata v12.0 (Stata Corporation, TX, USA) software. The PLR > 5 or NLR $<0.2$ indicated a high diagnostic ability of predictive model. SROC curve was also established, and diagnostic ability was considered as good if the area under the curve (AUC) was $>80 \%$. The heterogeneity was assessed based on the values of the inconsistency index (12). $12>$ $50 \%$ was considered as a significant heterogeneity. The meta-regression was performed to detect the source of heterogeneity. The subgroup analyses were performed based on the points of heterogeneity. Publication bias was assessed via Deeks' funnel, and we consider that $P<0.05$ to be indicative of publication bias. 
Strategy of data synthesis: The sensitivity, specificity, positive likelihood ratio (PLR), negative likelihood ratio (NLR), diagnostic score, and summary receiver operating characteristic (SROC) curve were pooled by the Stata v12.0 (Stata Corporation, TX, USA) software. The PLR $>5$ or NLR $<0.2$ indicated a high diagnostic ability of predictive model. SROC curve was also established, and diagnostic ability was considered as good if the area under the curve (AUC) was $>\mathbf{8 0} \%$. The heterogeneity was assessed based on the values of the inconsistency index (12). $12>50 \%$ was considered as a significant heterogeneity. The meta-regression was performed to detect the source of heterogeneity. The subgroup analyses were performed based on the points of heterogeneity. Publication bias was assessed via Deeks' funnel, and we consider that $P<0.05$ to be indicative of publication bias.

Subgroup analysis: The subgroup analyses were performed based on the different reference standards, and blind situations.

Sensitivity analysis: None.

Country(ies) involved: China.

Keywords: SPN, predictive model.

Contributions of each author:

Author 1 - Tian Bai.

Author 2 - Li-Juan Wen.

Author 3 - Na Zhang. 